\title{
Maximum Cross Section Reduction Ratio of Billet in a Single Wire Forming Pass Based on Unified Strength Theory
}

\author{
Xiaowei $\mathrm{Li}^{1,2, \mathrm{a}}$ \\ ${ }^{1}$ School of Civil Engineering, Panzhihua University, Panzhihua 617000, China \\ ${ }^{2}$ Department of Tunnel and Underground Engineering, \\ Southwest Jiaotong University, Chengdu, 610031 China \\ ailixxxwww@126.com
}

\begin{abstract}
Keywords: Unified Strength Theory, Reduction Ratio, Wire Drawing, Wire Extrusion
Abstract. For obtaining the maximum cross section reduction ratio (MCSRR) for any billet, the equation for estimating the MCSRR of billet in a single wire forming pass was obtained based on unified strength theory. It is proved that the equation for the MCSRR of billet in wire drawing pass is similar to that in a single wire extrusion pass. Included the contribution of both intermediate principal shear stress and varying tension-compression-ratio (TCR) to material mechanical property, the equation developed can reasonably apply to a variety of materials. If the TCR of the material is equal to 1 , the MCSRR of billet in a single wire forming pass is 0.63 . In addition, the ranges of the MCSRR of billet for both ductile and brittle materials in a single wire forming pass are obtained.
\end{abstract}

\section{Introduction}

Wire forming is classified as wire drawing and extrusion, and treated as three-dimensional axisymmetric problem in analysis. Wire drawing is a metalworking process used to reduce the cross-section of a wire by pulling the wire through a single, or series of, die(s). Although similar during process, drawing is different from extrusion, because during drawing process the wire is pulled, rather than pushed, through the die. Drawing is usually performed at room temperature, thus classified as a cold working process. For simplicity, suppose that [1]: the inner wall of conical die is smooth; the radial direction of plastic flow is toward the virtual apex of the inner wall of conical die; and the material of billet is rigorous-plastic. Moreover, basically, identical strength in tension and compression for material is adapted by investigators, which will result in considerably errors for practical purpose, for, pulled or extruded through conical die, material of billet will develop Bauschinger effect, and the TCR of ductile metal varies from 0.77 to 1 , but is not always equal to 1 .

Ever since the unified strength theory was presented, researchers have produced fruitful works in many fields [2,3]. Assuming the yield surfaces are convex, the unified solution for the MCSRR of billet in a single wire forming pass is presented based on unified strength theory, which includes the contributions of both intermediate principle shear stress and the varying strength in tension and compression, and is suitable for both wire drawing and extrusion. It is proved that the previous solutions for billet with identical strength in tension and compression in a single wire drawing or extrusion pass are all special cases of the unified solution.

\section{Unified Strength Theory}

Based on twin shear strength theory, M.H. Yu [4] developed unified strength theory. The theory includes the contribution of $\sigma_{2}$ to material strength, and uses a uniform mechanical model to descript the plastic features of material. The mathematical expression for unified strength theory is simple and given as follows:

$$
F=\sigma_{1}-\frac{\alpha}{1+b}\left(b \sigma_{2}+\sigma_{3}\right)=\sigma_{t}, \quad\left(\sigma_{2} \leq \frac{\sigma_{1}+\alpha \sigma_{3}}{1+\alpha}\right) .
$$




$$
\begin{aligned}
& F=\frac{1}{1+b}\left(\sigma_{1}+b \sigma_{2}\right)-\alpha \sigma_{3}=\sigma_{t}, \quad\left(\sigma_{2} \geq \frac{\sigma_{1}+\alpha \sigma_{3}}{1+\alpha}\right) . \\
& b=\frac{(1+\alpha) \tau_{o}-\sigma_{t}}{\sigma_{t}-\tau_{o}}=\frac{(1+\alpha)-B}{B-1}, \quad \alpha=\sigma_{t} / \sigma_{c}, \quad B=\sigma_{t} / \tau_{o} .
\end{aligned}
$$

where, $\sigma_{t}$ is the tensile ultimate strength of a material, $\sigma_{c}$ the compressive ultimate strength, $\tau_{o}$ the shear ultimate strength, $\alpha$ the TCR, varying from 0.77 to 1.0 for ductile metal material, 0.33 to 0.77 for brittle metal material, and generally less than 0.5 for geotechnical materials, $B$ tension shear ratio, and $b$ participation factor of intermediate principal shear stress.

\section{Analysis on wire drawing for inward radial flow}

When wire is pulled through smooth conical die, radial flow is induced, pointing to the virtual apex of inner wall of conical die. The schematic diagram for steady-state axisymmetric problem, used to carry out the development of inward - radial -flow wire drawing, is plotted as Fig. 1.

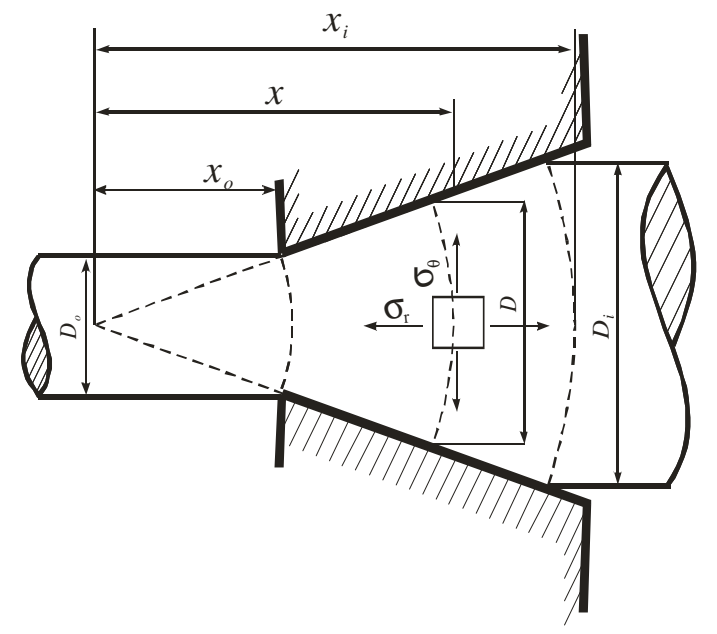

Fig. 1 Schematic Diagram for wire drawing

where $D_{o}$ is the diameter of billet at the die exit, $D_{i}$ at the die entry, and $D$ at any point within die; $x_{o}$ is the distance between the virtual apex of the inner wall of conical die and the die exit, $x_{i}$ the distance between that and the die entry, and $x$ the distance between that and any point within die; $\sigma_{r}$ is the positive direction of radial principal stress at any point within die, $\sigma_{\theta}$ the positive direction of the circumferential.

According to elasto-plastic theory, the relations for positive stresses on the surfaces of micro-body located in any point of billet within the region of die can be obtained as: $\sigma_{1}=\sigma_{r}>\sigma_{2}=\sigma_{3}=\sigma_{\theta}$. According to the equation, we can get: $\sigma_{r}-\sigma_{\theta}>0$. By the criterion condition of unified strength theory, one of Eqn. 1 and Eqn. 2 can be appropriately selected to be used to carry out analysis. Substituting both $\sigma_{r}-\sigma_{\theta}>0$ and $1 \geq \alpha>0$ into the criterion condition of Eqn.1, given as:

$$
\frac{\sigma_{1}+\alpha \sigma_{3}}{1+\alpha}-\sigma_{2}=\frac{\sigma_{r}+\alpha \sigma_{\theta}}{1+\alpha}-\sigma_{\theta}=\frac{\sigma_{r}-\sigma_{\theta}}{1+\alpha}>0 .
$$

According to Eqn.4, the Eqn.1 is used to analysis, substituting $\sigma_{l}=\sigma_{r}>\sigma_{2}=\sigma_{3}=\sigma_{\theta}$ into the Eqn.1, then, the following equation can be given as:

$$
\sigma_{\theta}=\frac{\sigma_{r}-\sigma_{t}}{\alpha} .
$$


From Eqn.5, it is obvious that the yield stress during wire drawing process is independent of the parameter factor of intermediate principle shear of $b$. According to elasto-plastic theory [5], the differential equation for three-dimensional axisymmetric problem can be obtained as:

$$
\frac{d \sigma_{r}}{d x}+\frac{2\left(\sigma_{r}-\sigma_{\theta}\right)}{x}=0
$$

Substituting Eqn.5 into Eqn.6, and give:

$$
\frac{\alpha x}{2} \frac{d \sigma_{r}}{d x}+(\alpha-1) \sigma_{r}+\sigma_{t}=0
$$

when $\alpha \neq 1$, Integrating Eqn.7, and the following equation can be got as:

$$
\frac{2(1-\alpha)}{\alpha} \ln |x|=\ln \left|\sigma_{r}-\frac{\sigma_{t}}{1-\alpha}\right|+c .
$$

Substituting both $x \geq 0$ and $\sigma_{r}-\sigma_{t} /(1-\alpha)<0$ into Eqn.8, and we can get the following equation as:

$$
\sigma_{r}=\frac{\sigma_{t}}{1-\alpha}-e^{-c} x^{\frac{2(1-\alpha)}{\alpha}} .
$$

\section{MCSRR of billet in a single wire drawing pass}

The integral constant in Eqn.9 can be determined by the stress conditions at die entry. Combining equation $x=x_{i}, \sigma_{r}=0$ with Eqn.9, $e^{-c}$ can be obtained as:

$$
e^{-c}=\frac{\sigma_{t}}{1-\alpha} x_{i}^{\frac{-2(1-\alpha)}{\alpha}}
$$

The radical principle stress expression can be obtained by substituting Eqn.10 into Eqn.9 as follow:

$$
\sigma_{r}=\frac{\sigma_{t}}{1-\alpha}\left(1-\left(\frac{x}{x_{i}}\right)^{\frac{2(1-\alpha)}{\alpha}}\right)
$$

At die exit, $x=x_{o}, D=D_{o}$; at die entry, $x=x_{i}, D=D_{i}$, owing to $x_{o} / x_{i}=D_{o} / D_{i}$, the radial principal stress expression at die exit can be obtained by substituting formula $x_{o} / x_{i}=D_{o} / D_{i}$ into Eqn.11 as follow:

$$
\sigma_{r o}=\frac{\sigma_{t}}{1-\alpha}\left(1-\left(\frac{D_{o}}{D_{i}}\right)^{\frac{2(1-\alpha)}{\alpha}}\right) .
$$

The radial principal stress, $\sigma_{r o}$, should be less than the tensile yield strength of material, $\sigma_{t}$. According to Eqn.12, the following equation can be then got as:

$$
\frac{\sigma_{t}}{1-\alpha}-e^{-c}\left(\frac{D_{o}}{D_{i}}\right)^{\frac{2(1-\alpha)}{\alpha}} \leq \sigma_{t} .
$$

According to Eqn.13, the following equation can be obtained as: 


$$
-\left(\frac{D_{o}}{D_{i}}\right)^{2} \leq-\alpha^{\left(\frac{\alpha}{1-\alpha}\right)} .
$$

The MCSSR of billet can be defined as: $R=1-D_{o} / D_{i}$, Then, the following equation can be got by substituting Eqn.14 into $R=1-D_{o} / D_{i}$ :

$$
R=1-\left(\frac{D_{o}}{D_{i}}\right)^{2} \leq 1-\alpha^{\left(\frac{\alpha}{1-\alpha}\right)}
$$

Using $\alpha=1$, and finding the limit of Eqn.15, the Eqn. 15 of the coefficient of MCSSR of billet based on Yu's unified strength theory can be changed into an equation based on Yu's unified yield criterion. It is found that the coefficient of MCSSR of billet in a single wire drawing pass based on Yu's unified yield criterion is a constant, which is 0.63 . It had been certified that the Mises and Tresca yield criterions are just special cases of Yu's unified yield criterion. Therefore, if the tension strength a material is equal to its compression strength, the MCSSR of billet in a single wire drawing pass is 0.63.

\section{MCSRR of billet in a single wire extrusion pass}

The principal stresses in billet during wire extrusion process meet $\sigma_{l}=\sigma_{r}>\sigma_{2}=\sigma_{3}=\sigma_{\theta}$. Therefore, the Eqn.9 is applicable for estimating the principal stress in billet during wire extrusion process. The integral constant in Eqn. 9 can be determined by the stress conditions of $x=x_{o}$ and $\sigma_{r}=0$ at die exit. Substituting $x=x_{o}$ and $\sigma_{r}=0$ into Eqn.9, and leading to:

$$
e^{-c}=\frac{\sigma_{t}}{1-\alpha} x_{o}^{\frac{-2(1-\alpha)}{\alpha}} .
$$

The radial principle stress expression can be obtained by substituting Eqn.16 into Eqn.9 as follow:

$$
\sigma_{r}=\frac{\sigma_{t}}{1-\alpha}\left(1-\left(\frac{x}{x_{o}}\right)^{\frac{2(1-\alpha)}{\alpha}}\right)
$$

The radial principal stress expression at die entry can be obtained by substituting $x_{o} / x_{i}=D_{o} / D_{i}$, into Eqn.17 as follow:

$$
\sigma_{r i}=\frac{\sigma_{t}}{1-\alpha}\left(1-\left(\frac{D_{i}}{D_{o}}\right)^{\frac{2(1-\alpha)}{\alpha}}\right) .
$$

The radial principal stress of $\sigma_{r i}$ should be no less than the tensile yield strength of material, $-\sigma_{c}$. According to Eqn.18, the following equation can then be got as:

$$
\frac{\alpha \sigma_{c}}{1-\alpha}\left(1-\left(\frac{D_{i}}{D_{o}}\right)^{\frac{2(1-\alpha)}{\alpha}}\right) \geq-\sigma_{c} .
$$

Rewriting Eqn.19, and, then Eqn.14 can be got again. Simultaneously, Eqn.15 can also be obtained. Thus it can be proved that the MCSRR of billet in a single wire drawing pass is equal to that in a single wire extrusion pass, supposing the die is similar. 


\section{Contributions of $\alpha$ to the MCSRR of billet in a single wire forming pass}

The curve of the maximum reduction, $R$ versus the TCR, $\alpha$, is shown in Fig. 2. Such following solutions can be showed from Fig. 2 as: $R$ increases with $\alpha$; when $\alpha$ increases to 1 , the value of $R$ is 0.63 ; if the billet in a single wire forming pass is brittle material, i.e., $\alpha$ varying from 0.33 to 0.77 , the relative range of the value of $R$ is $0.42-0.58$; if the billet in a single wire forming pass is ductile material, i.e., $\alpha$ varying from 0.77 to 1 , the relative range of the value of $R$ is $0.58-0.63$.

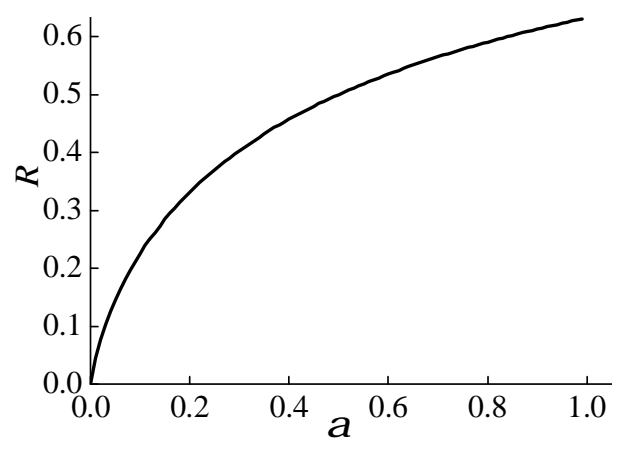

Fig.2 Relationship between $R$ and $\alpha$

\section{Conclusions}

The MCSRR of billet in a single wire drawing pass is equal to that in a single wire extrusion pass. If the tension strength of a billet's material is equal to its compression strength, the MCSRR of billet in a single wire forming process is 0.63 . If the billet is a brittle material, the relative range of the MCSRR of billet in a single wire forming process is $0.42-0.58$. If the billet is a ductile material, the relative range of the MCSRR of billet in a single wire forming process is $0.58-0.63$.

\section{Acknowledgements}

This work was financially supported by the Fund Program of Sichuan Province Key Lab for Comprehensive Development and Utilization of Industrial Solid Waste (SC_FQWLY201305, SC_FQWLY201403), Sichuan Education Department Fund Program (16ZB0475), and Panzhihua University Doctor Fund Program (bkqj2015004).

\section{References}

[1] R.A.C.Slater: Engineering Plasticity Theory and Application to Metal Forming Processes (Mechanical Press, China 1983). (in Chinese)

[2] M.H. Yu, S.Y. Yang, C.Y. Liu: China Civil Engineering Journal, Vol. 30 (1997) No.2, p.14. (in Chinese)

[3] J.H. Zhao: Strength Theory and Its Applications (Science Press, China 2003). (in Chinese)

[4] M.H. Yu: Unified Strength Theory and its Applications (Springer, Germany 2004).

[5] R. Wang, Z.H. Xiong, W.B. Huang: Foundation of Plastic Mechanics (Science Press, China 1982). (in Chinese) 